\title{
PermJoin: An Efficient Algorithm for Producing Early Results in Multi-join Query Plans
}

\author{
Justin J. Levandoski \\ Mohamed E. Khalefa \\ Mohamed F. Mokbel \\ Department of Computer Science and Engineering, University of Minnesota, Minneapolis, MN \\ \{justin,khalefa,mokbel@cs.umn.edu\}
}

\begin{abstract}
This paper introduces an efficient algorithm for Producing Early Results in Multi-join query plans (PermJoin, for short). While most previous research focuses only on the case of a single join operator, PermJoin takes a radical step by addressing query plans with multiple join operators. PermJoin is optimized to maximize the early overall throughput and to adapt to fluctuations in data arrival rates. PermJoin is a nonblocking operator that is capable of producing join results even if one or more data sources are blocked due to slow or bursty network behavior. Furthermore, PermJoin distinguishes itself from all previous techniques as it: (1) employs a new flushing policy to write in-memory data to disk, once memory allotment is exhausted, in a way that helps increase the probability of producing early result throughput in multi-join queries, and (2) employs a novel state manager module that adaptively switches operators between joining in-memory data and disk-resident data in order to maximize overall throughput.
\end{abstract}

\section{INTRODUCTION}

Traditional join algorithms (e.g., see [1], [2]) are designed with the implicit assumption that all input data is available beforehand. Furthermore, traditional join algorithms are optimized to produce the entire query result. Unfortunately, such algorithms are not suitable for emerging applications and environments that call for a new join algorithm design that is: (1) applicable in cases where input data is retrieved from remote sources through slow and bursty network connections and (2) optimized to produce early join results in a nonblocking manner while not sacrificing performance in processing the complete query result. Examples of such applications include web-based environments, where data is gathered from multiple remote sources and may exhibit slow and bursty behavior [3]. In addition, web users prefer early query feedback, rather than waiting an extended period for the complete result. Another vital example is scientific experimental simulation where experiments may take up to days to produce largescale results. In such a setting, a join query should be able to function while the experiment is running, and not have to wait for the experiment to finish. Also, scientists prefer to receive early feedback from long-running experiments in order to tell if the experiment must halt and be restarted due to unexpected results [4]. Other applications that call for new non-blocking join algorithms that produce early results include streaming

This work is supported in part by the Grant-in-Aid of Research, Artistry, and Scholarship, University of Minnesota, DTC Digital Technology Initiative Program, University of Minnesota, and DTC Intelligent Storage Consortium (DISC), University of Minnesota applications, workflow management, data integration, parallel databases, sensor networks, and moving object environments.

Toward the goal of maintaining high result throughput in emerging environments, several research efforts have been dedicated to the development of non-blocking join operators (e.g., see [3], [5], [6], [7], [8], [9], [10]). However, with the exception of [6], these algorithms focus on query plans containing a single join operator. The main focus of these algorithms is to optimize for high throughput locally at each operator. Optimizing for local throughput does not necessarily contribute to the goal of maximizing overall throughput in a multi-join query plan. Considering the complete query plan calls for a new set of optimization techniques that maximize early throughput in multi-join query plans.

In this paper, we propose a new efficient non-blocking join algorithm for Producing Early Results in Multi-Join query plans (PermJoin for short). PermJoin goes beyond the idea of single-operator non-blocking join algorithms and explores a holistic approach to optimization techniques for maximizing throughput in multi-join query plans in new and emerging environments. To this end, PermJoin exploits a set of techniques that considers all join operators in a query plan, rather than viewing each operator as a separate entity. PermJoin employs the symmetric hash join algorithm [10] to join incoming data in memory. Once runtime memory is exhausted, Permjoin intelligently selects a percentage of data to be flushed to disk based on expected query throughput contribution. Furthermore, PermJoin manages the state of each join operator, alternating between memory and disk processing, in order to maximize the throughput of the multi-join query plan. It is important to note that PermJoin is capable of producing complete and exact query results, making it suitable for applications that do not tolerate approximations.

PermJoin distinguishes itself from all other non-blocking join algorithms in two novel aspects: (1) PermJoin employs a novel adaptive memory flushing technique that is triggered once memory is full. Unlike previous flushing techniques for non-blocking join algorithms, the flushing technique in PermJoin maximizes the overall query throughput for a multijoin query plan by predicting the throughput contribution of in-memory data. (2) PermJoin employs a novel state manager module, that has the ability to switch any join operator back and forth between joining in-memory data and disk-resident data based on the operation most beneficial to the query throughput. Such a state manager module does not exist in previous non-blocking join algorithms. 


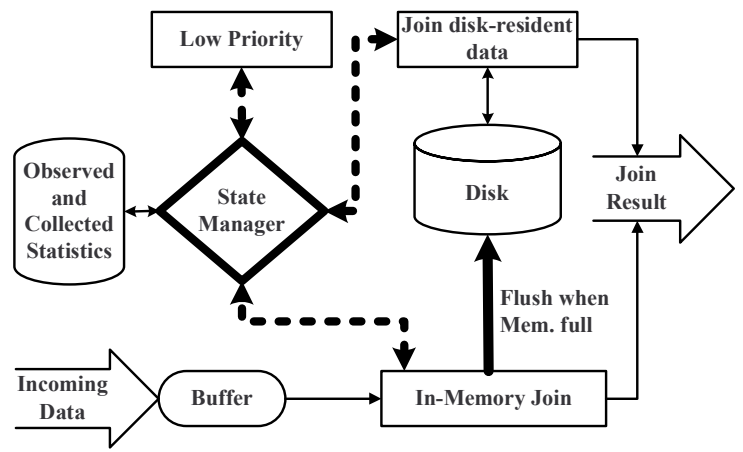

Fig. 1. Overview of the PermJoin Algorithm

\section{RELATED WORK}

The symmetric hash join [10] is the most widely used non-blocking join algorithm for producing early join results. However, it was designed for cases where all input data fits in memory. With the massive explosion of data sizes, several research attempts have aimed to extend the symmetric hash join to support disk-resident data [3], [5], [7], [8], [11]. These methods employ in-memory hash buckets to manage data. A percentage of these buckets are flushed to disk either individually [3], [8] or in groups [7], [9] when input data exhausts memory allocated for the query. All of these algorithms employ a symmetric hash join in order to achieve their non-blocking behavior. The main difference between them lies in the flushing algorithm used to free memory and maintain a high throughput. However, these techniques focus only on the case of a single join operator with no applicable extension for multi-join query plans. PermJoin employs a novel flushing algorithm that explicity considers the expected overall contribution of data for multiple join operators in a query plan to maximize throughput in real-time environments.

The closest work to PermJoin is the state-spilling method [6]; it is, to the authors' knowledge, the only work that addresses multi-join query plans. The basic idea behind state spilling is to score each hash partition group (i.e., pairs of corresponding hash buckets) in the query plan based on its past contribution to the query result. When memory is full, the partition group with the lowest score is flushed to disk. PermJoin distinguishes itself from this approach in two novel respects. First, PermJoin employs a novel flushing technique that takes into account both the input and output characteristics at each join operator. Using this information, the flushing algorithm predicts the contribution of data residing in the hash buckets. Second, PermJoin employs a novel state manager that switches each operator in the query plan between joining in-memory and disk-resident data in order to maximize the overall query throughput.

\section{Permuoin: An Overview}

This section gives an overview of the PermJoin algorithm, centered around the following two novel methods: (1) A new memory flushing algorithm, designed with the goal of evicting data from memory that will contribute to the result throughput the least, and optimized for overall (rather than local) early result throughput. (2) A state manager module designed with the goal of placing each operator in a state that will positively affect result throughput. Each operator can function in an inmemory, on-disk, or blocking state.

Figure 1 gives a state diagram for PermJoin with its novel aspects highlighted in bold. In this work, we consider left-deep query plans with $m$ binary join operators $(m>1)$, as depicted in Figure 2.As depicted in the diagram, whenever a new tuple $R_{S}$ is received by the input buffer from source $S$ of operator $O$, the state manager determines how the tuple is processed. If $O$ is currently not in memory, then $R_{S}$ will be temporarily stored in the buffer until $O$ is brought back to memory. Otherwise, $R_{S}$ will be immediately used to produce early results by joining it with in-memory data. Initially, all joins operate in memory. Once memory becomes full, PermJoin frees memory space by flushing a percentage of in-memory data to disk (depicted by the bold line from in-memory join to disk in Figure 1). The core of the PermJoin algorithm includes the state manager module (depicted by a bold diamond in Figure 1). The state manager is a continuously running thread that aims to place each join operator in one of three states (depicted by dotted bold lines): (1) Joining in-memory data, (2) Joining disk-resident data, or (3) Low Priority, i.e., producing results only if resources are available. At any time, the state manager may opt to switch a particular join operator from one state to another. In general, PermJoin consists of the following four components:

Memory Flushing. In PermJoin, the symmetric hash join [10] is used to produce early results in online environments. If memory allotment for the query plan is exhausted, data is flushed to disk to make room for new input, thus continuing the production of early results. We propose the AdaptiveGlobalFlush algorithm that aims to produce a high early overall throughput for multi-join query plans. The AdaptiveGlobalFlush policy flushes partition groups simultaneously (i.e., corresponding hash partitions from both hash tables). The main idea behind AdaptiveGlobalFlush is to consider partition groups across all join operators in concert, by iterating through all possible groups, scoring them based on their expected contribution to the overall result throughput, and finally flushing the partition group with the lowest score, i.e., the partition group that is expected to contribute to the overall result throughput the least.

To accomplish its goal, the AdaptiveGlobalFlush algorithm takes into account the following three characteristics of data in the query plan: (1) Global Contribution of the data, i.e., the ability to contribute to the overall throughput, (2) Data Arrival Patterns, i.e., changes in data arrival rates to the query plan, and (3) Data Properties, i.e., join attribute distribution or whether the data is sorted. A main drawback of previous flushing techniques is the inability to consider all three properties [6], [7], [8]. The AdaptiveGlobalFlush algorithm overcomes this drawback by considering all three properties. State Manager. The main responsibility of the state manager is to place each join operator in the most beneficial state in terms of maximizing the overall query throughput. As a motivating example for the state manager, consider the query pipeline in Figure 2(a) with four interconnected join operators: 


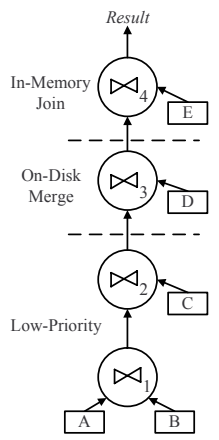

(a) State Example

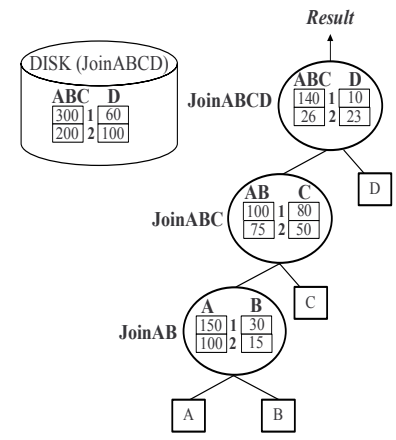

(b) Memory and Disk State
Fig. 2. Multi-Join Plan Examples

a base operator $J_{o i n}$ with inputs sources $A$ and $B$, an operator $J_{o i n}$ with inputs $A B$ (the output of $J_{o i n}$ ) and source $C$, an operator $J_{o i n}$ with inputs $A B C$ and source $D$, and a root operator $\mathrm{Join}_{4}$ with inputs $A B C D$ and source $E$. During query runtime, sources $A$ and $B$ may be transmitting data, while sources $C, D$, and $E$ are blocked. In this case, query results can only be generated from the base operator $J_{o i n}$. The overall query results produced by $J_{o i n}$ rely on the selectivity of the three operators above in the pipeline (i.e. Join 2 , Join 3 , and Join 4 ). If the selectivity of these operators is low, merging disk-resident data at either $\mathrm{Join}_{3}$ or $J_{\text {oin }}$ may be more beneficial in maximizing the overall query throughput than performing an in-memory join at the base operator Join $_{1}$. Thus, the state manager may decide to place $J_{\text {oin }_{4}}$ in the in-memory (i.e., default) state, Join 3 in the on-disk merge state, while Join 1 and Join 2 are placed in a low-priority state.

The state manager accomplishes its task by invoking a daemon process that traverses the query pipeline from top to bottom. During this traversal, it attempts to determine the operator $O$ closest to the root that will produce a higher overall throughput in the on-disk state compared to its in-memory state. If this operator $O$ exists, it is immediately directed to its on-disk merge state to process results. Meanwhile, all operators below $O$ in the pipeline are directed to temporary block while all operators above $O$ in the pipeline continue processing tuples in memory.

The design rationale behind the state manager is fundamentally different than that of the AdaptiveGlobalFlush. With AdaptiveGlobalFlush, the goal is to predict the least valuable in-memory data to flush to disk in order for the query to run effectively. Due to the changing nature of the query during runtime, data once determined to be the least beneficial by the flushing algorithm may turn out to be valuable later in runtime. For example, in Figure 2(b), the data on disk at Join $A B C D$ may be able to produce a higher result throughput than keeping all operators in their in-memory states. Thus, the rationale behind the state manager is to implement an efficient algorithm in order to find and use disk-resident data that becomes beneficial to the overall throughput.

In-Memory join. PermJoin employs the symmetric hash join algorithm to produce early join results [10]. Figure 3(a) gives the main idea of the symmetric hash join. Each input source

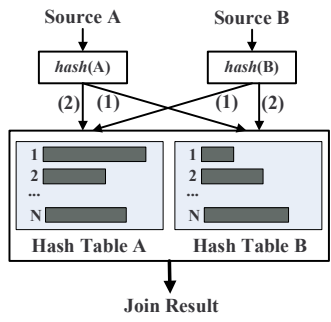

(a) Symmetric Hash Join

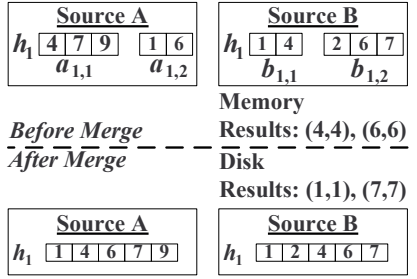

(b) On-Disk Merge

Fig. 3. In-Memory Join and On-Disk Merge

( $A$ and $B$ ) maintains a hash table with hash function $h$ and $n$ buckets. Once a tuple $r$ arrives from input $A$, its hash value $h(r)$ is used to probe the hash table of $B$ and produce join results. Then, $r$ is stored in the hash bucket $h\left(r_{A}\right)$ of source $A$. A similar scenario occurs when a tuple arrives at source $B$. Symmetric hash join is typical in many join algorithms optimized for early results [3], [7], [10].

On-disk join. PermJoin operators in the disk merge state employ a disk-based sort-merge join to produce results (e.g., see [6], [7]). Figure 3(b) gives an example of the sort-merge join in which partition group $h_{1}$ has been flushed twice. The first flush resulted in writing partitions $a_{1,1}$ and $b_{1,1}$ to disk while the second flush resulted in writing $a_{1,2}$ and $b_{1,2}$. Results from joining disk-resident data are produced by joining (and merging) $a_{1,1}$ with $b_{1,2}$ and $a_{1,2}$ with $b_{1,1}$. Partitions $\left(a_{1,1}, b_{1,1}\right)$ and $\left(a_{1,2}, b_{1,2}\right)$ do not need to be merged, as they were already joined while residing in memory; the results produced from in-memory and on-disk operations are labeled in Figure 3(b). Flushing partition groups and using a diskbased sort-merge has the major advantage of not requiring timestamps for removal of duplicate results [6], [7]. Thus, once a partition group is flushed to disk it is not used again for inmemory joins.

\section{REFERENCES}

[1] G. Graefe, "Query Evaluation Techniques for Large Databases," ACM Computing Surveys, vol. 25, no. 2, pp. 73-170, 1993.

[2] L. D. Shapiro, "Join Processing in Database Systems with Large Main Memories," TODS, vol. 11, no. 3, pp. 239-264, 1986.

[3] T. Urhan and M. J. Franklin, "XJoin: A Reactively-Scheduled Pipelined Join Operator," IEEE Data Engineering Bulletin, vol. 23, no. 2, pp. 27-33, 2000.

[4] G. Abdulla, T. Critchlow, and W. Arrighi, "Simulation Data as Data Streams," SIGMOD Record, vol. 33, no. 1, pp. 89-94, 2004.

[5] Z. G. Ives, D. Florescu, M. Friedman, A. Y. Levy, and D. S. Weld, "An Adaptive Query Execution System for Data Integration," in SIGMOD, 1999.

[6] B. Liu, Y. Zhu, and E. A. Rundensteiner, "Run-time operator state spilling for memory intensive long-running queries," in SIGMOD, 2006.

[7] M. F. Mokbel, M. Lu, and W. G. Aref, "Hash-Merge Join: A Nonblocking Join Algorithm for Producing Fast and Early Join Results," in ICDE, 2004.

[8] Y. Tao, M. L. Yiu, D. Papadias, M. Hadjieleftheriou, and N. Mamoulis, "RPJ: Producing Fast Join Results on Streams through Rate-based Optimization," in SIGMOD, 2005.

[9] S. Viglas, J. F. Naughton, and J. Burger, "Maximizing the Output Rate of Multi-Way Join Queries over Streaming Information Sources," in VLDB, 2003.

[10] A. N. Wilschut and P. M. G. Apers, "Dataflow Query Execution in a Parallel Main-Memory Environment," in PDIS, 1991.

[11] G. Luo, C. Ellmann, P. J. Haas, and J. F. Naughton, "A scalable hash ripple join algorithm," in SIGMOD, 2002. 